\title{
Water Content Determination
}

National Cancer Institute

\section{Source}

National Cancer Institute. Water Content Determination. NCI Thesaurus. Code C134116.

Determining the quantity of water contained in a material. 